\title{
Primeira Risposta de Niccolò TARTAGLIA A LUdovico FerRari - TraduÇão e TRANSCRIÇão
}

\author{
Fábio Maia Bertato \\ Centro de Lógica, Epistemologia e História da Ciência - CLE - Unicamp - Brasil
}

(aceito para publicação em agosto de 2016)

\section{Tradução ${ }^{1}$}

\section{RESPOSTA DADA POR NICCOLÒ TARTAGLIA \\ Bresciano, Professor das Matemáticas \\ em Veneza.}

Ao Messer Ludovico Ferrari, Leitor Público das ditas

Matemáticas em Milão, a uma sua petição, ou Car-

tello de disputa, a ele mandada no Mês de

\section{Fevereiro de 1547,}

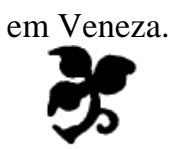

V. O. P. ${ }^{2}$

$[* * *]$

\footnotetext{
${ }^{1}$ Esta é a resposta (Contra-Cartello) de Niccolò Tartaglia ao primeiro Cartello de Ludovico Ferrari. A transcrição e tradução ao português de tal Cartello foi publicada em BERTATO, 2015. A tradução em inglês do mesmo Cartello pode ser encontrada em BERTATO, 2016. O leitor familiarizado com a língua italiana perceberá que apesar da tentativa de fidelidade da tradução, algumas correções e adaptações foram efetuadas, a fim de se capturar o sentido de algumas sentenças de Tartaglia, cujo estilo confuso e repetitivo foi duramente criticado por alguns de seus contemporâneos.

${ }^{2}$ O significado da sigla V.O.P. é desconhecido aos estudiosos da Disfida entre Ferrari e Tartaglia. Provavelmente, seja uma abreviação de vir optimus ("homem ótimo, excelente").
}

RBHM, Vol. 16, n 32, p. 101-112, 2016 


\section{| Ao Excelente Messer Ludovico Ferrari, Digníssimo Leitor Público das Matemáticas em Milão.}

Excelente $M$ [esser]. Ludovico, no dia 13 do presente, recebi um vosso Cartello, impresso em Milão no dia 10 do presente, o qual me entregou de vossa parte a Senhoria do messer Ottaviano Scotto, que me disse haver infinitos outros para serem enviados a toda Itália. Do mesmo modo, no fim do vosso Cartello, vós me avisastes de haver mandado cópias a diversos Senhores (que sabem das Matemáticas), em Roma, em Veneza, em Milão, em Florença, em Ferrara, em Bolonha, em Salerno, em Pádua, em Pavia, em Pisa e em Verona, e me haveis registrado o nome de cada um dos ditos Senhores no fim de vosso Cartello, os quais em suma são cinquenta e três. Assim o fizestes, crendo que com tal bravata me assustaria totalmente, mas estais redondamente enganado, porque vos afirmo e juro como verdadeiro cristão que, depois que nasci, aqui jamais ter havido nova que tanto me consolasse ou recreasse como essa. C Do vosso Cartello replicarei somente o seu principal conteúdo ou assunto, ao qual darei resposta resoluta, pois se eu quisesse registrar ou replicar a toda vossa injuriosa, caluniosa e mordente palavra dita naquele, teria de dar a cada uma, a devida e conveniente resposta, sendo assim necessário ocupar de escritura um caderno de cinco fólios e, como se sabe, todo escrito muito longo sói gerar confusão ou fastio aos leitores, coisa que no presente não me apraz. Reservo-me, porém, [o direito] de poder responder (a tais particularidades) toda vez que me pareça oportuno.

Para dar início ao que acima propus, digo que o verbo substancial do vosso dito Cartello é o seguinte. $\mathbb{C}$ Vós dizeis que eu disse, no último tratado de meu livro novamente impresso (intitulado Quesiti \& inventioni diverse), algumas caluniosas, injuriosas e mordentes palavras contra o Excelente Senhor Girolamo Cardano, médico milanês, no presente Leitor público em Pavia, e vos parecendo tal meu dizer ser muito desarrazoado, concluís dizendo que não somente para defender a verdade, mas também porque isso toca a vós por ser seu criado, haveis deliberado fazer publicamente conhecer o meu engano ou malignidade e, para alcançar o efeito dissestes ultimamente que vos oferecíeis para disputar publicamente comigo, em lugar igualmente cômodo, diante de juízes idôneos, amplamente em Geometria, Aritmética e em todas as disciplinas que delas dependam, como Astrologia, Música, Cosmografia, Perspectiva, Arquitetura e outras, bem como aceitais de disputar não somente sobre o que escreveram autores gre|gos, latinos e vulgares sobre tais faculdades, mas também sobre as minhas novas invenções, contanto que eu também semelhantemente aceite as vossas. E a fim de não me cansar ou enfastiar, vós oferecestes de jogar e depositar tanto dinheiro quanto me apeteça, até a soma de 200 escudos, etc.

À vossa proposta ou Cartello, respondo que as anteditas particularidades, por mim narradas ou registradas no meu dito livro, sobre o Excelente Senhor Girolamo Cardano, só foram anotadas por duas causas. Primeiro, para não faltar com minha palavra, ou seja, com a promessa feita à Sua Excelência com juramento (como se vê no fim do Quesito 34 da dita última parte do meu dito livro), pois, em verdade, não conheço infâmia que seja maior do 
que o perjúrio, não somente na nossa, mas em toda outra religião. $\mathbb{C}$ Segundo, as anotei com tais injuriosas e mordentes palavras para incitar Sua Excelência (e não vós) a escreverme qualquer coisa de sua mão, por haver muitas contas a saldar consigo, as quais, no presente, não me cabe o dever de narrá-los. Tal ardil também foi usado por Sua Excelência uma vez contra mim (como se vê na primeira carta a mim escrita por ele, no dia 12 de fevereiro de 1539, registrada no Quesito 32 do mesmo livro), quando escreveu que eu me demonstrei um grande ignorante com seu núncio livreiro, e que me julgava muito presunçoso, usando ainda muitas outras palavras caluniosas. Tais palavras foram empregadas, afirma Sua Excelência (em sua segunda carta), a fim de incitar-me a escreverlhe. Com isso, quero inferir que esses são certos tratos costumeiros entre nós, para nos incitarmos mutuamente a escrever. Digo, portanto, se fostes movido a escrever-me tal Cartello por vós mesmo, isto é, se não fostes impelido por Sua Excelência (coisa que não acredito), vos admoesto como irmão a tornar à vossa leitura e deixar tal empresa ao dito Excelente Senhor Girolamo, que é homem (como acredito que saibais), se for o caso de eu ter empregado alguma palavra desarrazoada contra ele, a ponto de ressentir-se. Mas, se por acaso Sua Excelência vos provocou a isso (como acredito), dizei a ele, de minha parte, que me escreva ou faça escrever segundo ele, e não segundo vós, isto é, em seu nome e não em vosso nome, pois se assim o fizer, lhe darei a resposta que a mim me pareça conveniente.

Podeis dizer que proceder assim agrada à Sua Excelência.

E eu vos respondo que proceder assim não agrada a mim, isto é, que não me apraz (no presente) responder a vós, seu criado, mas somente a ele, pois não tenho coisa alguma a resolver convosco, mas sim com ele.

Podeis também dizer que Sua Excelência não se encontra em Milão, e sim em Pavia.

Respondo-vos que nem eu me encontro atualmente em Bréscia, minha Pátria, mas me encontro em Veneza.

Podeis ainda dizer que Sua Excelência está ocupada da Leitura pública em | Pavia. C A isso vos respondo que se Sua Excelência está ocupada, também eu não estou ocioso. Pelo contrário, se Sua Excelência está ocupada com uma lição por dia, eu estou ocupado com mais de cinquenta. Não obstante, se necessário, posporei qualquer coisa, pois, em verdade, parece-me que as coisas que tratam da honra devem preceder a todas as outras.

Mas como não gostaria que belamente vos retirásseis do baile, no qual imprudentemente entrastes, vós dois juntos, ao afirmar que o Excelente Girolamo Cardano se encontra ocupado da leitura pública em Pavia, pondo com tal subterfúgio fim à festa por vós iniciada, e [tendo em vista que], como seu criado, convidei-o a disputar publicamente comigo para defender sua honra e ele recusou a partida, e que, todavia, desejo restar ao menos com esse pouco de honra, me pareceu conveniente cerrar-vos todos os passos, a fim de que não possais, nem ele, nem vós, sair da festa sem bailar. Portanto, novamente vos aviso de que, se por acaso, o dito Senhor Girolamo Cardano, todavia, tenha deliberado de não me escrever por saber (como prudente) de ter a culpa nesse assunto e de não ter causa alguma a lamentar de mim, mas somente dele e de seus ilícitos comportamentos contra mim, já seu grande amigo, vede ao menos de fazer com que ele se subscreva ao dito vosso Cartello de sua mão, bem como [que seja] seu companheiro em tal disputa, ao que assim fazendo, alegremente e com bom coração aceito a vossa grande oblação a mim feita, com 
vós e com ele junto, ou seja, de disputar com ambos largamente em Geometria, em Astronomia, Música, Cosmografia, Perspectiva, Arquitetura e outras, de acordo com o que vós sozinho me oferecestes em vosso Cartello. Não aceito, contudo, e não sou obrigado a aceitar, a vossa condição que consequentemente se interpõe, isto é, se acrescenta, ou seja, aquela parte em que dizeis que aceitais de disputar não somente sobre o que autores gregos, latinos e vulgares escreveram, mas também sobre as minhas invenções (ou seja, do meu dito livro) que tanto me deleitam, contanto que eu também aceite as vossas. Isso porque nenhum desafio pode ser condicionado, máxime com condição que seja prejuízo ou desfavor para o desafiado. Pelo contrário, todos os entendidos, que sobre os desafios escreveram, querem que todas as particularidades, que sejam de qualquer vantagem, restem nas mãos do desafiado, livres e francas.

Portanto, dessa tal vossa astuta condição ri muito e muito, pois vejo que com ela, haveis imaginado de atar-me assim o passo, de modo que, em tal disputa, eu não vos pudesse apresentar [nada] salvo o que escreveram os autores em tal faculdade (coisa ridícula!) ou ainda as coisas por mim postas e declaradas no meu livro, pensando firmemente que de tal particularidade eu não pudesse me aperceber. Todavia, nisso, por duas | causas vos reconheço como tolos. CPrimeiro, por crer que eu seja tão tolo, a ponto de que não pudesse compreender a rotundidade e a importância de tal vossa condição. Segundo, não vos percebeis, pobrezinhos, que com tais vossas condições, haveis descoberto, ou manifestado a mim e aos homens de engenho, a vileza de vossos corações e como temeis de entrar nessa festa por vós iniciada, o que é assaz importante.

Agora, para concluir, replico e digo alegremente que aceito a vossa larga oblação, [de disputar] convosco junto dele, mas não com a dita condição. Ao contrário, quero estar livre e franco para poder propor-vos, em tal disputa, aquilo que me apeteça nas ditas disciplinas ou nas dependentes, ou seja, [baseado] sobre algum autor ou afora todo autor, ou melhor, vos afirmo que muito evitarei não somente de não vos propor coisas postas pelos ditos autores, mas ainda de tal sorte que as coisas postas e disputadas pelos ditos autores não vos possam ajudar. Caso contrário, que grande tolice me reputaria ao propor-vos em tal caso alguma coisa posta pelos autores, se não vos acrescentasse alguma bela e engenhosa particularidade. Portanto, contentando-vos, a um e ao outro, com o que acima disse, subscrevo-me a vós ambos de própria mão, como mais acima foi dito. CMas, para alongar o assunto e finalmente reduzi-la a uma confusão que não tenha jamais cabo, vós poderíeis dizer (como haveis dito no vosso Cartello) que é necessário, antes que nos enfrentemos, determinar o lugar que seja igualmente cômodo em tal disputa.

Do mesmo modo, é necessário que encontremos e de acordo elejamos juízes idôneos, que devem ouvir e depois julgar mediante seu parecer.

É necessário ainda que vós determineis o preço que vos contentais de depor, ou de jogar, e a quem desejais depositá-lo, a fim de que eu esteja seguro de que, vencendo, haver o dito preço por vós deposto.

De modo semelhante, é necessário que vós exponhais as conclusões, dúvidas, casos ou questões que pretendeis de me propor em tal disputa, com os seus convenientes termos, para responder-vos segundo a sua quantidade e outras símiles particularidades.

CA fim de limpar, portanto, o caminho desses vossos agudos espinhos, com os quais vós pensastes de entreter-me durante a viagem, prolongando o assunto e finalmente (como 
acima dito), de reduzi-la totalmente em fumaça, responderei particularmente a cada uma das vossas ditas prorrogações e determinarei cada uma delas.

Digo, portanto, que para vosso mais comodíssimo lugar para realizar tal disputa, vos estabeleço Milão e a mim, Veneza. Caso contrário, vós podeis dizer como disputaremos nós dois juntos, estando vós em Veneza e eu em Milão; e como poderemos reunir os juízes, que | possam ouvir de viva voz a uma e a outra parte, já que é necessário que ambas as partes se encontrem em sua presença, de modo que possam ouvir de viva voz a nossas argumentações e conclusões. Vossa tal particularidade, ou condição, é um certo reduto, ou fortaleza, por vós astutamente fabricada, na qual esperáveis de vos salvar totalmente, ao dizer que jamais poderíamos convir nessas duas coisas, isto é, sobre o lugar onde havemos de disputar, ainda mais sobre a eleição dos juízes. E se, todavia, devesse eu convir, eu assim o faria em qualquer lugar privado, diante de quaisquer juízes amigos meus (por ter eu familiaridade com todos os homens peritos da Itália), para disputar em palavras sobre qualquer autor, ou ainda quaisquer autores (como lhes apeteça), sendo que tais juízes expediriam em uma só audiência, com pouca vergonha e nem honra de uma ou de outra parte, e assim poríamos fim à nossa querela. Mas, vos falhará o pensamento, porque deliberei que a nossa disputa seja (e permaneça) pública ao mundo, ou seja, quero que todos os nossos quesitos, casos ou questões (que em tal disputa se haverá de propor), sejam propostas públicas e impressas, ou estampadas, e do mesmo modo, as resoluções e respostas das mesmas, a fim de que todos os inteligentes do mundo possam comodamente ouvir, ver e fazer juízo da vossa e da minha qualidade. Isso porque, como deveis saber, para interrogações, quesitos ou questões matemáticas, raras vezes ocorre que se possa dar resolução imediata em [viva] voz (como se faz nas outras ciências, ou artes liberais), mas somente por escrito e com um tanto de tempo, porque assim ocorre na maior parte daquelas várias considerações, ações, cálculos e, às vezes, figuradas demonstrações. Nem mesmo nas resoluções daquelas calha muitos juízes, por estarem aquelas no primeiro grau de certeza, de tal modo que se as resoluções estiverem corretas e bem apresentadas, o adversário é obrigado a dever concedê-lo e, concedendo, não calha outros juízes. Mas, se vos parece [necessário] eleger juízes para a nossa disputa, eu elejo o mesmo vosso Excelente Senhor Girolamo Cardano juntamente convosco, de uma parte, e os inteligentes do mundo, de outra.

A respeito da disposição do dinheiro que pretendo jogar convosco, eu depositarei aqui em Veneza nas mãos da Senhoria do messer Ottaviano Scotto, vosso amigo, o qual assegurará a ratificação (sendo vós vencedores), de vos responder-lhes em Milão; o mesmo voto que fazeis vós.

A quantidade de dinheiro que pretendo depor e, similarmente, as questões que pretendo disputar, me reservo o direito de dizer quando da vinda de vossa resposta, a qual aguardo, por trinta dias após a apresentação desta. E aquela vindo como desejo, espero (com honestos modos) de lavar-vos otimamente a cabeça a ambos, de uma só vez, coisa que não saberia fazer nenhum barbeiro da Itália. Se nos ditos termos não me derdes outra resposta, deixarei aos inteligentes do mundo julgar a vossa qualidade, reservando-me, porém, a razão de poder proceder mais além, se assim me parecer oportuno. Dada em Veneza, 19 de fevereiro de 1547. | 
Eu, Niccolò Tartaglia Bresciano, Professor em Veneza das disciplinas matemáticas, afirmo o que acima eu disse.

Eu, Paulo Marescoro, sou testemunha do que está contido acima e de própria mão me subscrevi.

Eu, Mario Nizolio, sou testemunha do que está contido acima e de própria mão me subscrevi.

Eu, Tiberio Scardoa, sou testemunha do que está contido acima e de própria mão me subscrevi.

\section{Post-Scriptum}

A fim de que esta minha resposta não vos pareça muito privada, mandei imprimir mil [cópias] para mandá-las em geral por toda a Itália, já que é verdade que eu não pratiquei pelas cidades da dita Itália, nem pelos estúdios, onde se adquire a amizade e conhecimento dos homens peritos e doutos, assim como vós (porque, na verdade, a prática e a minha conversação sempre foram apenas no estúdio que tenho em meus aposentos, com meus discípulos). Posto que não somente não conto com a amizade como também não tenho conhecimento daqueles, não dirijo ou mando alguma das ditas minhas respostas a alguma particular pessoa douta, como fizestes vós, mas apenas as mando em geral a todos, nem quis mandá-las àqueles Excelentíssimos Senhores, por vós indicados no fim de vosso Cartello, pois me pareceu ser presunção grandíssima a minha fazê-lo, por não haver familiaridade nem conhecimento com algum deles, exceto dois deles: o Reverendo Senhor Gabriel Tadino, Prior de Barletta, que morreu há muitos meses, e o outro não desejo nomeá-lo por ainda mais respeito. Todavia, pensando que talvez de tal minha resposta teriam prazer (por entenderem tudo), me pareceu de dirigir-vos cinquenta e quatro [cópias] consignadas à Senhoria do messer Ottaviano Scotto, e lhe pedi que vos envie o mais rápido possível, das quais retenhais uma para vós e as outras cinquenta e três, mandeis uma a cada um dos ditos Senhores, se vos apetecer. Vale. 


\title{
Transcrição
}

\author{
RISPOSTA DATA DA NICOLÒ TARTALEA \\ Brifciano delle Mathematice Profeffore \\ in Venetia.
}

A Mefler Ludouico Ferraro delle dette Mathematice Lettor

Publico in Melano, d'vna fua rechiefta, ouer Cartello de difputa a lui mandata l'Anno

1547. del Mefe di Febraro

in Venetia.

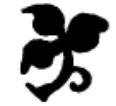

V. O. P.

$\left[\begin{array}{lll}* & * & *\end{array}\right]$

| Al Eccellente miffer Ludouico Ferraro delle Mathematice Lettor publico in Melano Digniflimo.

Eccellente M. Ludouico alli 13. del prefente riceueti vno voftro Cartello ftāpato in Melano alli 10. pur del prefente, qual me diede la Signoria de mefl. Ottauiano Scotto da parte voftra, $\&$ me diffe hauerne infiniti altri da mandare per tutta Italia. Et fimilmente voi in fine del detto v[oft]ro Cartello me auifati hauer mādata la coppia di quello a diuerfi Signori (che fanno delle Mathematice) in Roma, in Venetia, in Melano, in Firenze, in Ferrara, in Bologna, in Salerno, in Padoua, in Pauia, in Pifa, \& in Verona, \& me haueti regiftrato el nome di cadauno de detti Signori in fine del voltro Cartello, liquali in fumma fono 53. credendoui con tal voltra gran tagliata di fpauentarmi in tutto, ma ve fiti ingannato de groflo, perche vi affermo \& giuro da real Chriftiano, da poi ch'io nacqui in qua mai hauer hauuto noua che tanto mi confolaffe, ouer recreaffe quanto quefta. $\mathbb{C D}$ el qual voftro Cartello quiui replicaro folamente el fuo principal neruo, ouer foggetto, \& a quel daro rifoluta rifpofta, perche volendo io quiui regiftrare, ouer replicare ogni voftra ingiuriofa, caloniofa, \& mordente parola detta in quello, \& dapoi dare a cadauna di quelle, quella debita, \& conueniente rifpofta li fi conuegnaria, a me faria neceffario occupare de fcritura vn quinterno di carta, ogni troppo longa fcrittura, come fi fa, fol cumunamente generar confufione, ouer faftidiare li lettori di quella, laqualcofa per al prefente non mi piace. Referbādome pero di poter refpondere (a tai particolarita) ogni volta mi parera. 
Hor per dar principio a quello che di fopra ho propofto, Dico chel verbo foftantial de detto voftro Cartello e quefto. $\mathbb{C}$ Voi ditte chio ho detto nel vltimo trattato del mio libro nouamente ftāpato (intitolato Quefiti \& inuentioni diuerfe) alcune caloniofe, ingiuriofe, \& mordente parole, contra lo Eccellente Signor Hieronimo Cardano Medico Milanefe, \& al prefente Lettor publico in Pauia, \& parendoui tal mio dire, effer molto irragioneuole concludendo diceti, che non folamente per diffendere la verita, ma anchor perche quefto tocca a voi per effer fuo creato, haueti deliberato far publicamente conofcere il mio inganno, ouer malignita, \& per venire al effetto vltimamente diceti che offeritia a difputar publicamente con meco, in luoco equalmente commodo, \& dinanci a giudici idonei, largamente in Geometria, Arithmetica, \& in tutte le Difcipline che da quelle dipendono, come e Aftrologia, Mufica, Colmographia, Profpettiua, Architettura, \& altre, Et che accettati de difputar non folamente, fopra quanti Authori gre|ci, latini, \& volgari, che hanno fcritto in tal faculta, ma anchora fopra le mie noue inuentioni, pur che anchora io fimelmente accetti le voftre. Et accioche non me rincrefca la fatica, o fpefa ve offeriti di giucare, deponere quāti danari a me parera per fina alla fumma di 200. fcudi \&c.

Alla qual voftra propofta, ouer cartello rifpondo, che le predette particolarita, da me narrate, ouer regiftrate nel detto mio libro, del detto Eccellente Signor Hieronimo Cardano, Io ve le ho anotate, ouer regiftrate per due fol caufe, prima per non mancare della parola mia, cioe della promefla fatta a fua Eccellentia con giuramento (come appare in fine del Quefito 34. Della detta vltima parte del detto mio libro) perche inuero io non cognofco infamia che fia maggior del romper fede, \& non folamente nella noftra, ma in ogni altra religione. CSecondariamente ve le ho anotate, \& con tai caloniofe \& mordente parole per incitar fua Eccellentia ( \& non voi) a fcriuermi qualche cofa di fua mano, per hauer io molti conti da faldar con quella, liquali per al prefente non mi accade a douerli narrare, laqual cautella fu vfata anchor da fua Eccellentia vna volta verfo di me, (comme appare nella prima lettera a me fcritta da quella, l'anno 1539. a di 12. febraro, regiftrata nel Quefito 32, del detto mio libro) nella quale me fcriffe che io me era dimoftrato vn grande ignorante, con vn fuo nontio libraro, $\&$ che me giudicaua troppo profüptuofo cō moltre altre caloniofe parole, \& tai parole Sua Eccellentia (nella fecōda fua 1[ette]ra) afferma hauerle dette p[er] incitarmi a fcriuergli. Si che voglio inferire che quefti fono certi tratti coftumari tra noi per incitarfi lun con laltro a fcriuerci. E per tanto dico, fe ve feti mouefto a fcriuermi tal Cartello da voi ifteffo, cioe no fpinto da fua Eccellentia (cofa che non credo) ve admonifco da fratello a douer tendere alla voftra lettura, \& laffar tal imprefa al detto Eccellente Signor Hieronimo, elqual e huomo (come credo fapeti) che fe io hauero vfato alcuna parola irragioneuole contra di lui da refentirfene. Ma fe per cafo fua Eccellentia ve ha aprouocato a quefto (come credo) ditte a quella da parte mia che la mel fcriua, ouer faccia fcriuere secōdo lei, \& nō fecōdo voi, cioe in nome fuo, \& nō in nome v[oft]ro, il che facendo, io gli daro q[ue]lla rifposta che a me parera conueniēte.

$\mathrm{O}$ voi potrefti dire, che cofi piace di procedere a fua eccellentia.

Et io ve rifpondo che coli non piace di procedere a me, cioe chel non mi piace (per al prefente) de rifpondere a voi fuo creato ma folamēte a lui, per che io non ho daffare cofa alcuna con voi, ma fi con lui.

Potrefti anchor dire fua Eccellentia no fi troua al prefente in Melano, ma fi troua in Pauia. 
Ve rifpondo che nanche mi, non mi trouo al prefente in Brefcia Patria mia, ma mi trouo in Venetia.

Potrefti anchor dire foa Eccellentia fta occupata della Lettura publica in | Pauia. C A quefto ve rifpondo che fe fua Eccellentia fta occupata, Nanche io non fto ociofo:immo fe fua Eccellentia e occupata de vna lettione al giorno, \& io fon occupato de piu de 50 . \& nondimeno quando bifognara, pofponero ogni cofa, perche inuero quelle cofe doue fi tratta del honore, a me mi pare che debbeno precedere a tutte le altre.

Ma perche io non voria che bellamente ve cauafti fuora del ballo, nelqual feti īprudentemēte intrato, voi infieme cō lui, cō dire lo Eccellente Signor Hieronimo Cardano fi troua occupato della lettura publica in Pauia, \& io come fuo creato lho inuitato a difputar publicamente con meco per difender lhonor fuo \& lui ha riffutato el partito, e pero mene voglio reftar con quefto puoco di honore, \& con tal cautella puor fine alla fefta per voi principiata. Me apparfo de ferarue tutti i palli, accioche non poffiati, ne lui, ne voi vfcir della fefta fenza ballare. E per tanto di nouo ve auifo che fe per cafo el detto Signor Hieronimo Cardano haueffe pur deliberato de non volermi fcriuere per conofcerfi (come prudente) hauer il torto, in quefta cofa \& non hauer caufa alcuna a lamentarfi di me, ma folamente de lui \& de fuoi illiciti deportamenti vfati verfo di me gia fuo grande amico. Vedeti almen di far che lui fe fottofcriua al detto voltro Cartello di fua mano fi come voftro compagno in tal difputa ilche facendo alegramente, $\&$ con buon cuore accetto la voftra larga oblatione a me fatta cō voi, \& con lui infieme, cioe de difputare con ambidui largamente in Geometria, in Aftronomia, Mufica, Cofmographia, Profpettiua, Architettura, \& altre fi come voi folo me ve feti offerto nel voftro Cartello, vero e ch'io non accetto, ne manco fon tenuto di accettare quella voftra conditione che confequentemente ve interponeti, ouer agiongeti, cioe quella parte doue che diceti che accettati de difputare non folamente fopra quanti Autori, Greci, Latini, \& volgari, che in tai faculta habbiano fcritto, ma anchora fopra le mie noue inuētioni, (cioe del detto mio libro) che tanto me dilettano. Domente che io anchora accetti le voftre. Perche niuna desfida puo effer conditionata, \& mallime con conditione che fia in preiuditio, ouer in disfauor del disfidato, anci che tutti li intelligenti, che fopra alle desfide hanno fcritto, vogliono, che tutte le particularita, che fiano di qualche auantaggio reftino, nelle mani del disfidato libere, e franche.

E per tanto di quefta tal voltra aftuta conditione mene fon ridefto molto \& molto. perche vedo che con quella vi haueui immaginato de volermi legare a quefto paffo, che io non ve poteffe preponere in tal difputa faluo che li Autori che in tal faculta hanno fcritto (cofa ridiculofa) oueramente delle cofe da me pofte, \& dechiarate nel mio libro, \& penfauate fermamente che di tal particularita non mene douefle auedere: Ma in quefto per due | caufe vi conofco alquanto tondi. CPrima credere che io fia tanto tondo, che non douefre comprendere la rotondita di tal voftra conditione, \& la importantia di quella, Secondariamente non ve auertiti pouereti, che con tai voftra conditione, haueti fcoperto, ouer manifeftato a me, \& alli huomini de ingegno, la vilta di voftri cuori, \& come ve temeti a intrar in quefta fefta per voi principiata, il che importa affai.

Hor per venire alla conclufione replico \& dico che alegramente accetto la voftra larga oblatione con voi infieme con lui, ma non gia con la detta cōditione, anci voglio effer libero, e franco di poter proporui in tal difputa quello che a me parera, nelle dette difcipline, ouer dependente, o fia fopra ad alcun Autore, o fuora de cadaun Autore, anci vi affermo che 
molto mi guardaro non folamente da non preponerui cofe pofte dalli detti Autori, ma anchora di tal forte che per le cofe pofte, $\&$ difputate dalli detti Autori non ve pofciate agiutare, Anci che gran fciocchezza reputaria la mia apreponerui in tal cafo alcuna cofa pofta dalli Autori fe non vi gli agiongeffe qualche bella, \& ingeniofa particolarita. E per tanto contentandoui luno, e laltro di quanto ho detto di fopra, fottofcriuetiue ambi dui di propria mano come di fopra fu detto, $\mathbb{C}$ Ma per slongar la cofa $\&$ finalmente ridurla in vna confufione de non venir' giamai a vn capo, voi potrefti dire (come haueti detto nel voltro Cartello) bifogna prima nanti che vegniamo alle prefe, che determinamo el luoco che fia equalmente comodo in tal difputa.

Simelmente bifogna che trouiamo, \& che dacordo ellegemo Giudici idonei, che ne habbia ad aldire, \& dapoi giudicare il parer loro.

Eglie neceffario anchora che voi determinati el precio che ve contentati da deponere, ouer giocare, \& a chi il voleti depofitare, accio che io fia ficuro vincendo di hauer el detto precio da voi depofto.

Et fimelmente bifogna che voi dati fuora, le Conclufioni, Dubii, Casi, ouer Queftioni che pretendeti da propormi in tal difputatione con li fuoi conueniente termini da refponderui fecondo la quantita de quelli, \& altre fimile particolarita.

CPer anetarme adunque la via da quefte voftre acute fpine, con lequale voi haueui penfato da intertenermi per viaggio, \& di andar prolongando la cofa \& finalmente (come di fopra ho detto) di redurla totalmente in fumme. Rifpondaro particolarmente a cadauna de dete voltre proroghe \& determinaro cadauna di quelle.

Dico adunque che per voftro piu comodiflimo luoco da far tal difputa vi affegno Milano, \& a me Venetia. O voi potrefti dire, come difputaremo noi infieme ftando voi a Venetia, \& io a Melano, Come potremo noi redur li giuduci, che poffano al dire viua voce luna parte \& laltra, e pero eglie neceffario che ambe le parte fi trouano a fua prefentia talmente che | poffano dal dire viua voce le noftre Argumentationi, \& Conclufioni. La qual voltra particolarita, ouer conditione, e vn certo ridutto, ouer fortezza, da voi aftutamente fabricata, nella qual fperauate al tutto di faluarui, con dire giamai in quefte due cofe fe potremo conuegnire, cioe del luoco doue che hauemo da difputare, ma piu nella ellettione di giudici. Et fe pur me douero conuegnire, io tiraro coftui in qualche priuato luoco dauanti a qualche giudici amici mei (per hauer io famigliarita con tutti li periti huomini de Italia) a difputar in parole fopra qualche Autore, ouer piu Autori (come gli parera) li qual giudici ne fpedirano in vna audientia fola, con puoca vergogna, manco honore de luna \& laltra parte, $\&$ cofi porremo fine alla noftra querella. Ma el vi falira el penfiero, perche ho deliberato che tal noftra difputta fia (\& refti) publica al mundo, cioe voglio che tutti li noftri, Quefiti, Cafi, ouer Queftioni, (che in tal difputa fe hauera da proporre) fiano propofte publice \& impreffe, ouer ftampate, \& fimelmente le refolutioni, \& rifpofte di quelle, accioche tutti li inteligenti del mundo le poffano commodamente al dire, vedere, \& far giuditio della voltra, \& mia qualita. Perche doueti pur fapere che alle interrogationi, Quefiti, ouer Queftioni Mathematice, rare volte accade che vi fe poffa dar refolutione immediate in voce, (come fe fa nelle altre fcientie, ouer arti liberale) ma folamente in fcritto \& con alquanto di tempo, perche vi occorre in la maggior parte di quelle varie confiderationi, attioni, Calculationi, \& alle volte figurate demoftrationi, ne manco nelle refolutioni di quelle vi accade molti giudici, per effer quelle nel primo grado di certezza, talmente che ferai refolutioni farano 
vere, $\&$ ben rafolte lo auerfario e aftretto adouerle concedere, $\&$ concedendo le non vi accade altramente giudice, ma fe pur vi pare chel fi elleggia giudici fopra a tal noftra difputa, io elleggio, lo medefimo voftro Eccellente Signor Hieronimo Cardano infieme con voi da vna parte, \& li intelligenti del mondo da laltra.

Circa alla depofitione delli danari che pretendaro de giocar con voi io li depolitaro in mane della Signoria de meffer Ottauiano Scotto amico voftro qua in Venetia, qual promettara de rato, (reftando voi vencitori) de refponderueli in Melano, il medefimo voro che fati voi.

La quantita delli danari che io pretendo da deponere, \& fimilmente le queftioni che pretendo da difputare me referbo a dirlo, per fin alla venuta della voftra rispofta, qual afpetto per giorni 30, doppo la prefentatione, di quefta. Et quella venendo come defiderio, fpero (con honefti modi) di lauarue ottimamente, el capo ad ambidui in vn colpo folom cofa che nō fapria fare alcun barbier de Italia, \& fe nel detto termine non me dareti altra rifpofta laffero far giuditio alli intelligenti del Mondo della qualita voftra, Referbandome pero ragione di poter procedere piu oltra fe cofi mi parra di fare. Data in Venetia alli 19. di Febraro. 1547.

Io Nicolo Tartalea Brifciano, Delle Difcipline Mathematice in Venetia Profeffore, affermo quanto di fopra ho detto.

Io Paulo Marefcoro son teftimonio di quanto fi contien di fopra \& di man propria mi ho fottofcritto.

Io Mario Nizolio fono teftimonio di quanto di fopra fi contiene \& pero di mano mi fono fottofcritto.

Io Tiberio Scardoa fon teftimonio a quanto di fopra fi contiene e di man propria mi fono fottofcritto.

Da poi fcritta.

Accioche quefta mia rifpofta non vi paia molto priuata ne ho fatto imprimere 1000. per mandarne anchora io generalmente per tutta Italia, vero e che per no hauer io praticado per le Citta de detta Italia ne per li ftudii, doue fe acquifta la amicitia, \& cognitione delli huomini periti \& dotti, fi come voi (perche in vero la pratica; \& conuerfation mia fi e fempre ftata folamente per il ftudio, qual ho nella mia camera, \& con li mei Difcipuli,) Per il che non folamente manco della amicitia ma anchora della cognitione de quegli, E pero non derizzo, ouer, mando alcuna delle dette mie rifpofte ad alcuna particolar perfona dotta, come che haueti fatto voi, ma folamente le mando in general a tutti, ne manco non ho volefto mandare a quelli Eccellentiflimi Signori da voi anotati in fine del detto voftro Cartello, perche a me mi parea effer prefumption grandiffima la mia, a mandarui vna tal cofa non hauendo, non folamente famigliarita, ma cognitione de alcun de quelli, eccetto che de dui foli quali, luno fu il Reuerendo Signor Gabriel Tadino Prior di Barletta, qual e morto gia molti mefi, latro non voglio nominarlo altramente per piu rifpetti. Ma pur penfando che forli 
de tal mia rifpofta ne haueriano appiacere (per intendere il tutto) me apparfo de drizzaruene a voi 54. quale ho confignate alla Signoria de meffer Ottauiano Scotto, \& lho pregato che velle manda, piu prefto che fia pollibile, delle quale ne tenereti vna per voi, \& delle altre 53. ne mandareti vna a cadauno de detti Signori, piacendoui pero. Vale.

\section{Referências}

BERTATO, Fábio M. Primeiro Cartello di Matematica Disfida de Ludovico Ferrari a Nicolò Tartaglia - Tradução e Transcrição. Revista Brasileira de História da Matemática. Vol. 15, n. 30, pp. 95-100, 2015.

BERTATO, Fábio M. First Cartello di Matematica Disfida from Ludovico Ferrari to Niccolò Tartaglia - Translation and Transcription. Revista Brasileira de História da Matemática. Vol. 15, n. 31, pp. 131-136, 2016.

GIORDANI, Enrico. I sei cartelli di matematica disfida primamente intorno alla generale risoluzione delle equazioni cubiche di Lodovico Ferrari coi sei contro-cartelli in risposta di Nicolò Tartaglia comprendenti le soluzioni de' questi dall' una e dall' atra parte proposti. Milano: Ronchi, 1876.

Fábio Maia Bertato

Centro de Lógica, Epistemologia e História da

Ciência - CLE - Unicamp - Brasil

E-mail: fmbertato@cle.unicamp.br 